\title{
Description of Lyme disease-like syndrome in Brazil. Is it a new tick borne disease or Lyme disease variation?
}

\section{E. Mantovani ${ }^{1}$, I.P. Costa ${ }^{3}$, \\ G. Gauditano ${ }^{1}$, \\ V.L.N. Bonoldi ${ }^{1}$, \\ M.L. Higuchi ${ }^{2}$ \\ and N.H. Yoshinari ${ }^{1}$}

\author{
1Disciplina de Reumatologia, 2Departamento de Anatomia Patológica, \\ Instituto do Coração, Faculdade de Medicina, Universidade de São Paulo, \\ São Paulo, SP, Brasil \\ ${ }^{3}$ Departamento de Clínica Médica, Universidade Federal de Mato Grosso do Sul, \\ Mato Grosso do Sul, MS, Brasil
}

Correspondence

N.H. Yoshinari

Departamento de Reumatologia

Faculdade de Medicina, USP

Av. Dr. Arnaldo, 455

3o andar, sala 3139

01246-903 São Paulo, SP

Brasil

Fax: + 55-11-3061-7490

E-mail: lyme@lim17.fm.usp.br

Research supported by FAPESP

$\ldots \ldots \ldots \ldots \ldots \ldots$

Received May 16, 2006 Accepted December 18, 2006

\section{Abstract}

An emerging clinical entity that reproduces clinical manifestations similar to those observed in Lyme disease (LD) has been recently under discussion in Brazil. Due to etiological and laboratory particularities it is named LD-like syndrome or LD imitator syndrome. The condition is considered to be a zoonosis transmitted by ticks of the genus Amblyomma, possibly caused by interaction of multiple fastidious microorganisms originating a protean clinical picture, including neurological, osteoarticular and erythema migrans-like lesions. When peripheral blood of patients with LD-like syndrome is viewed under a dark-field microscope, mobile uncultivable spirochete-like bacteria are observed. PCR carried out with specific or conservative primers to recognize Borrelia burgdorferi sensu stricto or the genus Borrelia has been negative in ticks and in biological samples. Two different procedures, respectively involving hematoxylin and eosin staining of cerebrospinal fluid and electron microscopy analysis of blood, have revealed spirochetes not belonging to the genera Borrelia, Leptospira or Treponema. Surprisingly, co-infection with microorganisms resembling Mycoplasma and Chlamydia was observed on one occasion by electron microscopy analysis. We discuss here the possible existence of a new tick-borne disease in Brazil imitating LD, except for a higher frequency of recurrence episodes observed along prolonged clinical follow-up.

\section{Introduction}

Lyme disease (LD) was first described by Allen C. Steere in 1977, in Connecticut, USA, and defined as an infectious disease caused by the spirochete Borrelia burgdorferi and transmitted through the bite of Ixo-
Key words

- Lyme disease-like syndrome

- Tick-borne disease

- Mycoplasma

- Chlamydia

- Spirochete

- Lyme disease des scapularis ticks (deer ticks) (1). The geographical distribution of this disease is the northern hemisphere. The bacterium has not been isolated in South America, Africa or Australia.

In Brazil, for more than 15 years of research, a new syndrome that reproduces clini- 
cal manifestations similar to those of LD has been identified and has been called LD-like syndrome (LDLS) or LD imitator syndrome (LDIS). The term syndrome indicates that eventually other microorganisms alone or associated with spirochetes could reproduce the complete clinical picture observed in LD, including the typical skin lesion named erythema migrans.

Our laboratory, located in the Hospital das Clínicas, Faculdade de Medicina, Universidade de São Paulo (LIM-17), is the only public institution in Brazil accepting patients with suspected LDLS in order to perform serological exams and to coordinate research on LDLS. Monthly, about 150 blood or cerebrospinal fluid (CSF) samples sent from all the Brazilian States are examined and submitted to serology for the detection of B. burgdorferi antibodies. Since the disease is part of the differential diagnosis of several clinical syndromes, knowledge of it is very important for many medical specialties, mainly neurology (Mantovani E, Livramento JA, Apóstolos S, Yoshinari NH, unpublished data). This subject is of interest for veterinarians since the disease also affects domestic animals (2).

\section{Epidemiology}

Epidemiological inquiry is of great help to diagnose LDLS. Patients become ill after visiting wooded areas and/or areas with the presence of wild and domestic animals, especially those located in coastal or rural regions. Epidemiological data have shown that $50 \%$ of the patients report a history of tick bite or have had close contact with these parasites, such as finding a tick on their clothing or on animals or soil of the visited areas. Veterinarians, geologists, botanists, fishermen, and hikers are more predisposed to contracting the infection since they frequently enter the ecosystems where ticks and bacterium-contaminated animals are found $(3,4)$.
Studies carried out in risk areas, i.e., those with confirmed clinical LDLS cases, have shown that different wild animals such as small rodents, marsupials, and possibly other larger animals, participate as "reservoirs", since spirochetes can be visualized in the peripheral blood of these animals by dark-field microscopy (3-5). Uncultivable spirochetes have also been observed in the peripheral blood of cattle with arthritis and opossums (6). However, immobile spirochete-like structures, considered to be artifacts, viewed in prolonged culture of biological samples in Barbour-Stoenner-Kelly (BSK) medium can cause misdiagnosis of the presence of live bacteria. Electron microscopic analysis of these structures revealed that they were composed of microorganism flagella (Marzagão $\mathrm{G}$, unpublished data).

Ticks of the Ixodes loricatus (Neumann), Amblyomma cajennense (Fabricius) and Amblyomma aureolatum (Pallas) species are the usual vectors collected from wild animals in areas where the disease occurs in Brazil $(7,8)$. It is believed that vectors of the genus Ixodes are responsible for the transmission of this zoonotic disease among wild animals, since the Brazilian species have not been reported in humans, whereas Amblyomma ticks would be the potential transmitters of the disease to humans due to their voracity (7). In Brazil, there have been reports of accidental bites by A. cajennense causing erythema migrans and systemic complications. Furthermore, Costa et al. $(4,9)$ identified cases of LDLS patients associated with A. cajennense ticks in Campo Grande county, State of Mato Grosso do Sul, a geographic region where arthropods of the genus Ixodes were not collected during the studies described above.

\section{Background of the study of Lyme disease-like syndrome in Brazil}

The proposal to discover LD in Brazil 
arose in 1989 as a suggestion of Dr. Allen C. Steere since Ixodid ticks were present in Brazil (10). The initial multidisciplinary research group consisted of Professors Domingos Baggio (an entomologist from ICB, USP), Paulo Yasuda (a microbiologist from ICB, USP) and Natalino H. Yoshinari (a physician from FM, USP). At that time, LD was an unknown subject among Brazilian physicians, and therefore important dissemination work was planned involving Brazilian Health Institutions.

Some patients with skin lesions suggestive of Lyme borreliosis, without systemic symptoms or serological confirmation have been reported (11). Thus, in an attempt to understand this zoonosis in Brazil, the Laboratory for LD research was created in the Discipline of Rheumatology, Faculdade de Medicina, USP, sponsored by grants from Fundação de Amparo à Pesquisa do Estado de São Paulo (FAPESP) (10).

In 1992, two brothers were found to have acquired the disease in a condominium located in Cotia county, State of São Paulo (12). The boys had been bitten by ticks and developed erythema migrans, arthritis and general symptoms, with positive serology for B. burgdorferi. They received antibiotic therapy and their condition improved with no secondary complications. After the identification of other cases, it was observed that the clinical and laboratory profile of the disease in our country differed from the one observed in Europe, North America and Asia $(2,5,8,9)$.

Among the main differences, it was observed that the microorganism could not be cultivated or isolated when the usual culture medium for Borrelia, BSK, was employed. Also, serology applied to Brazilian patients by ELISA and Western blotting methods showed low reactivity when $B$. burgdorferi sensu stricto strain G39/40 antigens of North American origin were used, and antibody titers decreased rapidly during follow-up.

PCR applied to the sera and to other biological samples such as skin biopsies was negative when conservative primers that recognize the genus Borrelia or specific primers for the identification of B. burgdorferi sensu lato and sensu stricto were employed (9). Finally, from a clinical point of view, despite the presence of all the clinical manifestations of classic LD, the Brazilian disease differed by the high frequency of recurrence, especially in patients that had received delayed therapy during the initial phase of the disease (13).

The differences described suggested the existence of a unique infectious disease in Brazil that was similar to LD, but with an unclear etiology, which initially received the denomination of Brazilian Lyme-like disease $(2,4,5,9,14)$. Currently, we have chosen the denomination of LDLS or LDIS due to the presence of clinical manifestations similar to those found in LD, which imitate this zoonosis, but are caused by microorganisms that do not belong to the B. burgdorferi sensu lato complex. It is not North American LD-like disease (15) either, since this denomination is reserved for the disease observed in the South of the US, caused by a spirochete named B. lonestari (16). This spirochete, recently cultured in vitro (17), is transmitted by the Amblyomma americanum (Linnaeus) tick, promoting skin lesions that resemble erythema migrans without the development of systemic clinical complications, with serology being negative for $B$. burgdorferi.

Nonetheless, despite all of these particular features, there is some laboratory evidence suggesting that unidentified spirochetes participate as the etiological agent in Brazil due to the development of humoral $(10,12,13,18)$ and cellular (Bonoldi VLN, Gauditano G, Pirana S, Yoshinari NH, unpublished data) immune responses to antigenic components of $B$. burgdorferi sensu lato in Brazilian patients despite a lower sensitivity in comparison to those observed in the US and Europe. 
Figure 1. Microorganisms (arrow) visualized in patients with Lyme disease-like syndrome in Brazil (Dark-field microscope, 1000X, magnification bar $=10$ $\mu \mathrm{m})$.

Figure 2. Microorganisms (arrow) in the peripheral blood of a patient with Lyme disease-like syndrome (Panoptic staining, 1000X, magnification bar $=10$ $\mu \mathrm{m})$.

Figure 3. Cerebrospinal fluid of a patient with Lyme disease-like syndrome, with visualization of spirochetes (arrow; HE, 1000X, magnification bar $=10 \mu \mathrm{m}$ ).
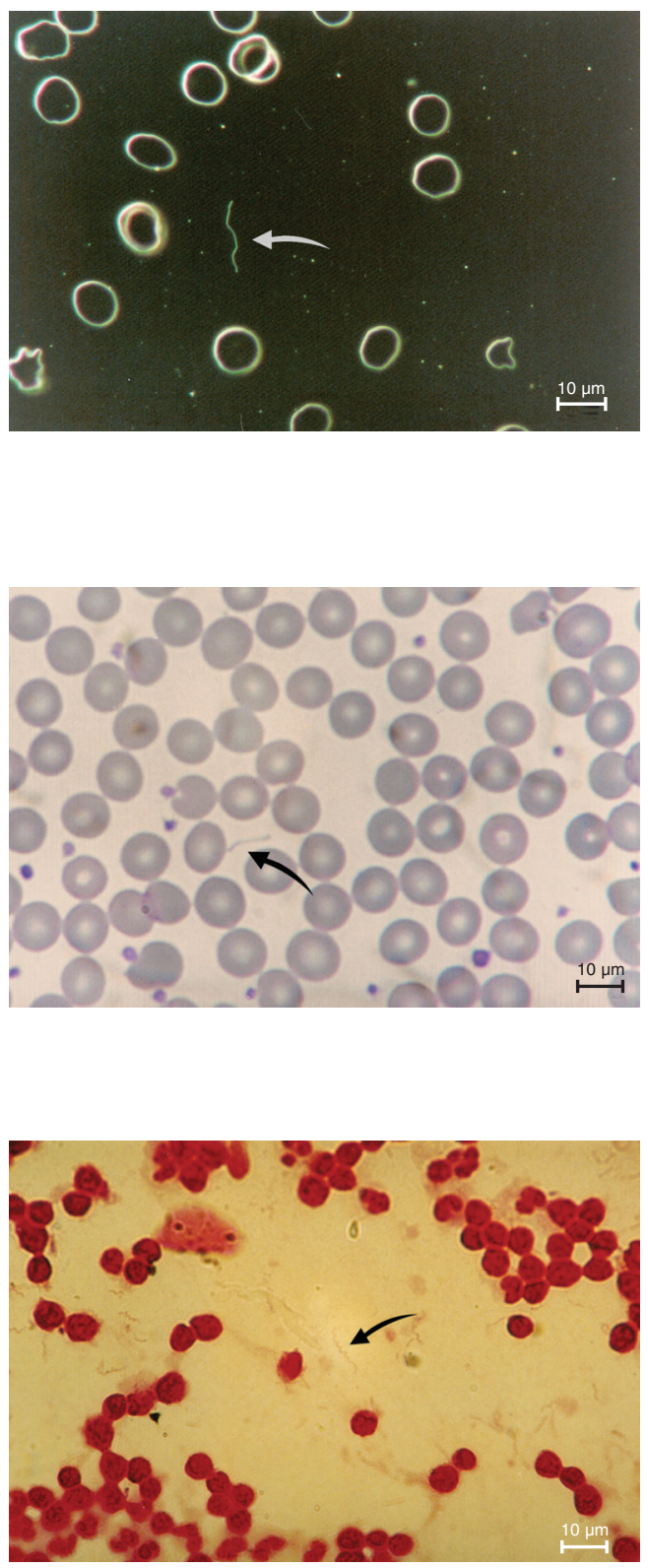

Figure 4. Peripheral blood of a patient with Lyme disease-like syndrome examined by electron microscopy (4200X): (A) Chlamydia; (B) Mycoplasma; (C) Spirochete (Prof. Maria de Lourdes Higuchi, Pathology Laboratory, INCOR). Magnification bar $=$ $500 \mathrm{~nm}$.

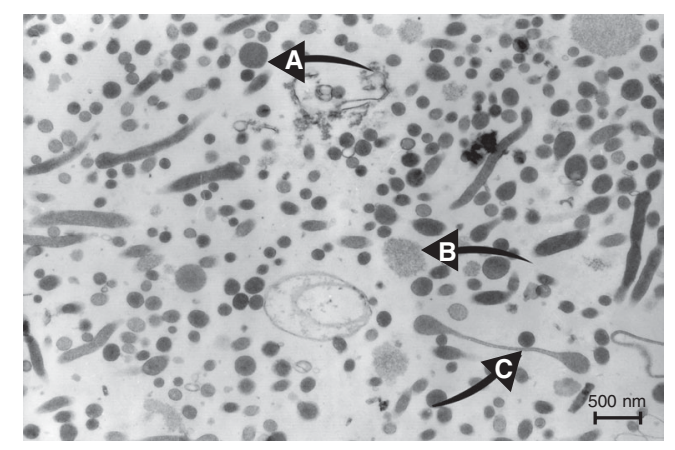

Furthermore, blood samples from LDLS patients examined under a dark-field microscope or submitted to Giemsa or Panoptic staining reveal the presence of spirochetelike microorganisms (Figures 1 and 2). Also, it was possible to identify corkscrew-shaped bacteria by hematoxylin-eosin staining in CSF from a patient with lymphomonocytic meningitis. This patient presented negative serology for syphilis, negative serology and PCR for leptospirosis, and positive serology for $B$. burgdorferi, with negative PCR for the genus Borrelia (Figure 3). In all cases, it was impossible to replicate bacteria seeded onto BSK medium.

Histological analysis of erythema migrans has revealed the presence of spirochetes (19). Melo et al. (19) performed a histopathological study of 31 patients with erythema migrans diagnosed in Manaus, Amazonas State, and identified spirochetes in one case $(3.22 \%)$ using Warthin-Starry silver stain. However, when modified WarthinStarry silver stain at $\mathrm{pH} 4.0$ (pyrocatechol) was used, no positive result was found among the 31 cases investigated.

Recently, electron microscopy carried out in the Pathology Laboratory of Instituto do Coração, Faculdade de Medicina, Universidade de São Paulo, on a peripheral blood sample from a patient with LDLS revealed the presence of a large amount of spirochetelike structures viewed by dark-field microscopy. Surprisingly, three types of bacteria were identified: Mycoplasma, Chlamydia and elongated structures suggestive of spirochetes (Figure 4).

\section{Is Lyme disease-like syndrome a new tick-borne disease?}

The finding of Mycoplasma, Chlamydia and unidentified spirochetes in the peripheral blood of a woman with a diagnosis of LDLS is an isolated description, but a very important result since it responds to many of our questions. Thus, this preliminary dis- 
covery indicates the existence of a clinical entity that imitates LD, characterized by the presence of a clinical picture resembling LD, but distinguished by a high incidence of relapsing episodes. Furthermore, the presence of unidentified spirochetes possibly not belonging to the genus Borrelia, among other fastidious microorganisms, explains the clinical similarities observed with LD found in the Northern hemisphere, as well as the weak immunologic cross-reactivity with $B$. burgdorferi. New investigations are in progress, but the idea that LDLS could be triggered by unidentified spirochetes associated with other fastidious microorganisms is fascinating and must be considered, since this hypothesis explains most of the questions raised in the past 15 years.

The medical literature points out that certain forms of reactive arthritis are associated with infections caused by intracellular survivor microorganisms of slow growth and difficult isolation and cultivation. This pattern of reactive arthritis is observed during infections by Chlamydia, Yersinia, Mycoplasma, and Borrelia. During the acute dissemination stage, microorganisms can be lodged in the articular cavity, as well as inside cells such as monocytes $(20,21)$ and a small number can survive in a "vegetative" state, being able to replicate intermittently in a process triggered by unknown mechanisms (22-25). This aspect has been clearly demonstrated for $C$. trachomatis infections, which persist in the form of atypical reticular bodies $(26,27)$.

The recurrence of clinical manifestations is a distinctive characteristic of infections by Chlamydia. The inability of the host organism to completely eliminate the pathogen leads to a chronic infection state, which can cause tissue damage sequelae. Thus, the infection, dissemination and development of immunity against Chlamydophila pneumoniae seem to have an important role in several diseases such as reactive arthritis, atherosclerosis, Alzheimer disease, and mul- tiple sclerosis $(28,29)$. A possible role of coinfection by M. pneumoniae and C. pneumoniae has been associated with the progress of atherosclerosis (29). Mycoplasma induces immunological changes in the host defenses that favor growth of other microorganisms (30). Lower ratios of $M$. pneumoniae/C. pneumoniae favor inflammation and instabilization of atherosclerotic lesions (31). The capacity of $C$. pneumoniae to infect and disseminate through several human cells after respiratory infection, such as epithelial, endothelial and smooth muscle cells, as well as macrophages, monocytes and lymphocytes, is well documented. Also, macrophage infection can lead to systemic dissemination, including the nervous system (28).

Unilateral facial nerve paralysis has been occasionally attributed to infectious agents, including herpes, mumps, rubella, influenza, syncytial respiratory virus, HIV, Coxsackie virus, B. burgdorferi, M. pneumoniae, $C$. pneumoniae, and the $B$-streptococcus group (32).

Among healthy individuals, some species of Mycoplasma are part of the normal human flora present on the surface of the oral, gastric or urogenital mucosa, and when certain species of Mycoplasma invade tissues, blood and organs, they are capable of inducing a complex systemic infection (33).

Increasing evidence suggesting that $M y$ coplasma can have an important role in the triggering or progression of rheumatoid arthritis has been recently reported. It has been proposed that Mycoplasma interacts nonspecifically with B-lymphocytes by interfering in immunity modulation and autoimmune reactions and promoting rheumatic disease (34). M. pneumoniae, M. salivarium and Ureaplasma urealyticum have also been found in articular tissue of patients with rheumatologic diseases, suggesting a possible pathogenic role of these infections (34).

When peripheral blood leukocytes of patients with rheumatoid arthritis were examined, approximately half of these patients 
were found to be infected with several $M y$ coplasma species. The most common species isolated was $M$. fermentans, followed by $M$. pneumoniae and $M$. hominis and finally, M. penetrans $(34,35)$. Specimens of $M$. fermentans were also found at high frequency $(88 \%)$ in samples of synovial fluid from patients with rheumatoid arthritis (36).

Recently, Eskow et al. (37) described 7 patients with osteoarticular manifestations resembling fibromyalgia symptoms caused by $M$. fermentans, transmitted by the bite of Ixodes ticks.

Other studies have shown that a high proportion of patients with chronic fatigue present infection by at least one species of $\mathrm{Myco}$ plasma: M. hominis, M. pneumoniae and $M$. fermentans $(28,33)$. It was also reported that patients with chronic fatigue demonstrated evidence of infection by $C$. pneumoniae and active human herpes virus-6 (28).

Certain bacteria have constituents that present strong homology to the host proteins (examples: YopH of $Y$. pseudotuberculosis and CD45, M. fermentans and CD4). This molecular mimicry can induce host tolerance against some microorganisms, promoting dysfunction of the immune system alertness (38).

Thus, recent publications incriminating microorganisms of intracellular survival as the agents responsible for articular or neurological complications, clinical features usually present in LDLS, support our view that there is a new emerging syndrome in Brazil caused by co-infection with these fastidious microorganisms. Based on the clinical presentation of LDLS, existence of serological cross-reactivity with $B$. burgdorferi and visualization of spirochete-like structures in peripheral blood and CSF viewed by microscopic analysis, we assume that unidentified spirochetes not belonging to the genus Borrelia must be the obligatory infectious agents present among other latent microorganisms.

In North America and Europe, information on LD is increasing fast, and certainly new concepts will be presented in the future with the objective of clarifying the microbiological, clinical and laboratory differences observed on different continents. There have been descriptions of the simultaneous presence of Borrelia and Chlamydia in the synovium of patients with Lyme arthritis (39). Also, concurrent infection of the central nervous system by $B$. burgdorferi and Bartonella henselae has been recently described, representing a novel tick-borne disease complex $(40,41)$.

For these reasons, we believe that many new answers are forthcoming concerning tick-borne diseases, including co-infections and participation of latent microorganisms in the changing pathogenesis and prognosis of LD or LDLS. Certainly, these new investigations will contribute to the understanding of questions such as time of treatment, choice of antibiotics, explanation of the high incidence of post-Lyme syndrome, fatigue and chronic fatigue, even in patients who have been treated. Also, many controversies concerning understanding LD such as the presence of relapsing episodes, disease chronicity, indication of antibiotic therapy in the late stage of the disease and development of autoimmunity, aspects of LD that still baffle researchers, could be explained if in fact coinfection of spirochetes with other latent microorganisms exists.

\section{Clinical picture of Lyme disease-like syndrome}

The diagnosis of LDLS is based on epidemiological and clinical aspects. Since the etiological agent has not been isolated or cultivated in Brazil, false-positive serology for B. burgdorferi is a helpful laboratory parameter.

Like classical LD, LDLS develops in stages and the distinctive aspect of its early phase is a skin lesion closely similar to that of classical erythema migrans. Three to 32 days after a tick bite, a red macula or papula appears in some patients, that usually ex- 
pands to form a large annular erythematous lesion, commonly with a red border and a lighter central part (Figure 5).

The lesion is neither painful nor pruriginous, is rarely vesiculated and can be present as other forms such as homogeneously erythematous or multi-dotted forms which tend to coalesce.

In the early blood dissemination phase of LDLS, new multiple and less extensive lesions can sometimes appear and are called secondary annular lesions, like those observed in LD. Erythema migrans is observed mainly in $70 \%$ of cases in the US, and in $50 \%$ of cases in Europe. In Brazil, recent unpublished studies (Shinjo SK, Marchiori P, Gauditano G, Costa IP, Oliveira ACP, Bonoldi VLN, Mantovani E, Yoshinari NH) have revealed that $43.3 \%$ of 30 patients in whom LDLS started with neurological involvement presented skin lesions. In another report, Costa et al. (9) identified skin manifestations in 8 of 16 patients (50\%) from Campo Grande, Mato Grosso do Sul State. The primary erythema migrans-like lesion, when present, appears on average 10.8 days after the tick bite and lasts 3 to 90 days, with a mean duration of 30 days $(2,5,8)$.

Associated with erythema migrans, general flu-like signs and symptoms appear, corresponding to the systemic dissemination of the microorganisms, such as fever, chills, headache, neck pain, myalgia, articular pain, dysesthesia of extremities, lymphadenopathy, conjunctivitis, malar rash, and sleep alterations.

When not treated adequately in its primary phase, LDLS can develop with secondary complications. In this late dissemination stage, new skin lesions, named recurrent erythema migrans, can appear alone or followed by systemic complaints. They are similar to the initial lesion, but usually are multiple and less extensive, and appear far from the original bite site (2).

In the secondary stage of LDLS new presentations of skin lesions have been re- ported in Brazil, such as lymphocytoma (Figure 6), scleroderma-like lesions that imitate acrodermatitis chronica atrophicans (Figure 7), and panniculitis (Figure 8) (14).
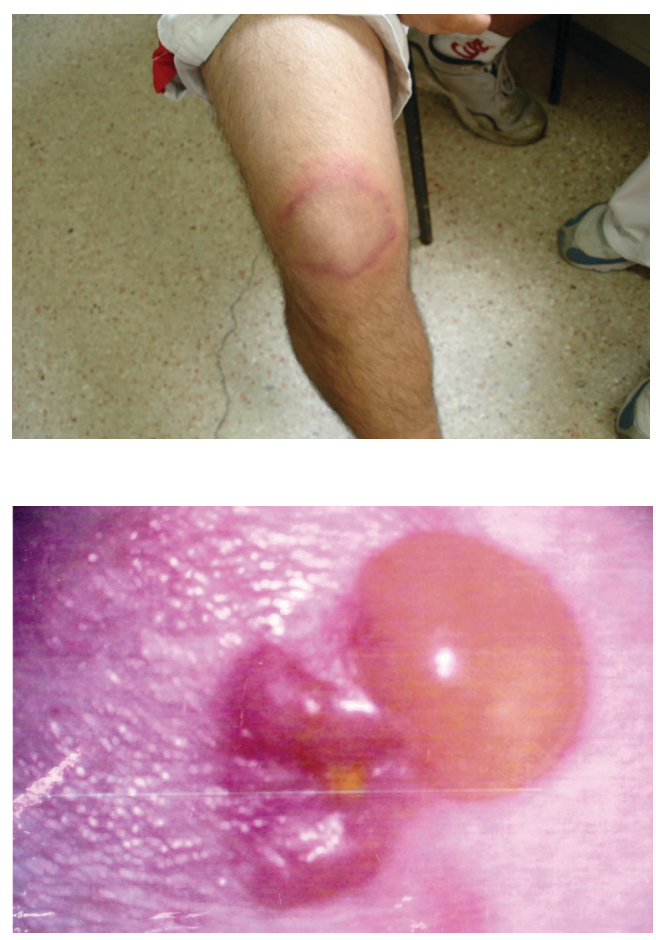

Figure 6. Blister lesion in ankle following tick bite episode. Histopathological analysis compatible with benign lymphocytoma.

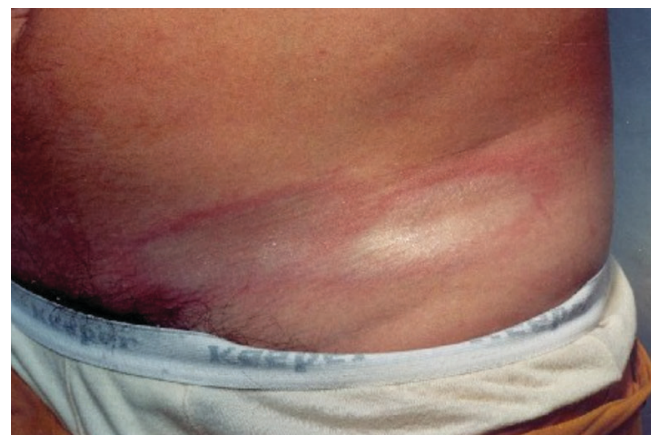

Figure 7. Patient with scleroderma-like lesion at the tick bite site.

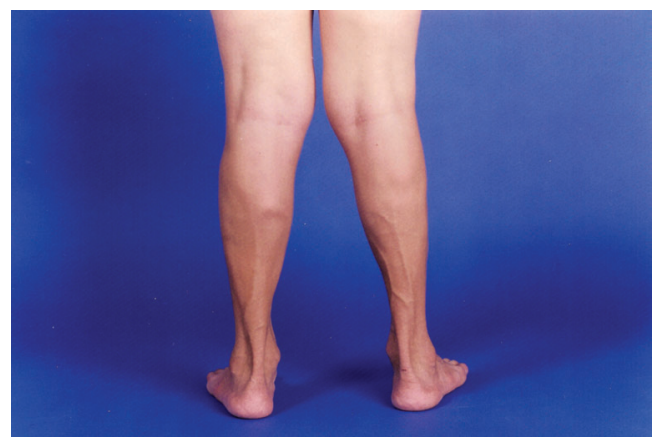

Figure 5. Acute skin lesion at the tick bite site, characterized by expansive erythematosus halo. 
Involvement of systemic organs in LDLS can be estimated on the basis of two series studied in two different Brazilian States. Yoshinari et al. $(5,8)$ studied 57 patients with LDLS mostly from São Paulo State and detected neurological symptoms in $31.5 \%$, arthritis in $35.2 \%$, arthralgia in $40.3 \%$, chronic fatigue in $8.7 \%$, and cardiac disease in only $5.2 \%$. In another series, Costa et al. (9) described 16 patients from Mato Grosso do Sul State and detected arthritis in $25 \%$, neurological symptoms in $37.5 \%$, and myalgia in $31.2 \%$.

In LDLS, arthritis can appear weeks, months, or years after the initial tick bite, and can be accompanied or not by skin lesions or neurological manifestations. The study of the synovial fluid reveals an inflammatory process with a predominance of neutrophils. The arthritis is initially mono- or oligoarticular, involving large joints, especially the knee, in 70 to $80 \%$ of cases, with the duration of the inflammatory flare ranging from days to weeks, generally with spontaneous regression even in the absence of specific treatment (2).

When patients are not treated with antibiotic therapy they can develop recurrences, and the tendency is the appearance of progressively longer flares and an increasing number of joints involved, including small joints, sometimes with symmetry and morning stiffness, simulating rheumatoid arthritis.

In Brazil, arthritis, usually of the knee, occurs in approximately $30 \%$ of cases in the early disseminated stage, less than 3 months from disease onset, frequently associated with complaints of myalgia or chronic fatigue. The occurrence of arthritis in the late dissemination stage, 3 months after the onset of the disease, affects roughly half of the patients with LDLS, who tend to present polyarticular and symmetrical joint involvement. Myositis is a rare complication which was observed in two of 30 patients $(6.7 \%)$ with neurological manifestation of LDLS, and the diagnosis must be confirmed by increased enzyme levels, electromyographic alteration and a muscle biopsy. Cardiologic involvement in LDLS has been a rare finding (about $5 \%$ of the cases studied) and has been diagnosed in patients with cardiomegaly and arrhythmia (2).

The neurological manifestations of LD are present in 15 to $20 \%$ of patients in the US and are characterized by the triad meningitis, cranial neuritis and sensory or motor peripheral radiculoneuritis.

The neurological involvement occurring in LDLS is similar to that observed in LD. Recently, we studied 30 patients with neurological involvement due to LDLS (Shinjo SK, Marchiori P, Gauditano G, Costa IP, Oliveira ACP, Bonoldi VLN, Mantovani E, Yoshinari NH, unpublished results), 19 of whom had been clinically followed over a mean period of 42.8 months. The following neurological symptoms were observed: meningismus in $15(50 \%)$, peripheral motor radiculitis in $12(40 \%)$, peripheral sensitive radiculitis in $10(33.3 \%)$, tendon hyporeflexia in $5(16.7 \%)$, ocular symptoms in $11(36.7 \%)$, peripheral facial nerve palsy in $8(26.7 \%)$, hearing disturbance in 3 (10\%), dysphagia in $2(6.7 \%)$, dysarthria in $2(6.7 \%)$, loss of equilibrium in $1(3.3 \%)$, psychiatric disease in $6(20 \%)$, cognitive disturbances in 8 $(26.7 \%)$, myalgia in $3(10 \%)$, muscle fasciculation in $1(3,3 \%)$, seizures in $2(6.7 \%)$, ataxic gait in $3(10 \%)$, loss of anal or bladder sphincter control in $2(6.7 \%)$, and coma in 1 (3.3\%).

Pirana et al. (42) showed that 39 of 200 serum samples (19.5\%) from patients with peripheral facial nerve palsy had positive serological reactivity against $B$. burgdorferi. In another study, Lorenzi et al. (43) described the presence of antibodies to this spirochete in $23.3 \%$ of 47 consecutive patients with sudden deafness enrolled in the study, emphasizing the diagnosis of LDLS in Brazilian patients with otolaryngological manifestations. However, the hearing out- 
come of treated Borrelia-seropositive patients was similar to that of seronegative subjects.

Recently, Sato et al. (44) described the first patient with suddenly diminished vision as a consequence of bilateral papilledema and corneal keratitis, who responded to antibiotic treatment. Our experience with 30 neurological patients with LDLS demonstrated that cranial neuritis was present in $43.3 \%$, and ocular symptoms were identified in $36.7 \%$, with involvement of the optic, oculomotor, trochlear, and abducens cranial nerves. Facial nerve palsy was the second most frequent manifestation, affecting $23.3 \%$ of the patients.

Tertiary or late complications of LD occur years after the initial contagion, and are characterized by the development of chronic arthritis, by skin lesions called chronic atrophic acrodermatitis, found more often in Europe and associated with infection by $B$. afzelii, and by neurological alterations similar to those of chronic degenerative neuropathies. These chronic changes are difficult to diagnose worldwide since autoimmune phenomena are often present in these clinical conditions, interfering with the serology for the identification of antibodies against $B$. burgdorferi sensu stricto.

Acrodermatitis chronica atrophicans is a skin lesion of delayed onset similar to scleroderma, observed more often in Europe, and its evolution is divided into two phases. In the inflammatory phase, there are erythematous nodules or plaques with a lighter center, which develop in the distal extremities of the limbs and are generally bilateral. After weeks or months, in the second phase, the skin has an atrophic aspect, similar to "cigarette paper", with local hypo- or hyperpigmentation, sometimes with a squamous aspect, accompanied by pain, pruritus and paresthesia (45).

In Brazil, some patients develop plaque skin changes at the tick bite site resembling scleroderma. Histological analysis of these cases reveals the presence of vasculitis with infiltration by mononuclear cells and collagen deposition in the dermis. The lesions last a long time and usually do not respond to antibiotic treatment. The macroscopic and microscopic aspects of these lesions are similar to those of acrodermatitis chronica atrophicans (9).

\section{Laboratory aspects}

In the US, Western blotting is the most sensitive and specific technique used for the laboratory diagnosis of $\mathrm{LD}$, with almost $50 \%$ positivity for acute cases and $80 \%$ positivity during convalescence (46). The sera of US patients with $L D$ recognize specific antigens of $B$. burgdorferi sensu stricto, allowing the standardization of Western blotting results. The assay for IgM class antibodies is considered positive for LD in the presence of specific bands of the following molecular masses: 18, 21, 28, 37, 41, 45, 58, and 93 $\mathrm{kDa}$, and is considered positive for the $\mathrm{IgG}$ class in the presence of bands of 18, 21, 28, $30,39,41,45,58,66$, and $93 \mathrm{kDa}$ (47). In the US, Western blotting is defined as positive if 5 of the $\operatorname{IgG}$ bands or two of the $\operatorname{IgM}$ bands shown above are found (48).

However, in Brazil, due to the fact that the etiological agent of spirochetes of LDLS does not behave as if it belonged to the B. burgdorferi sensu lato complex, patient sera exhibit a different pattern of reactivity upon Western blotting analysis when B. burgdorferi sensu stricto $\mathrm{G} 39 / 40$ of North American origin is used, as observed in our Laboratory.

This explains why the sera of patients with LDLS, when sent to the US for Western blotting analysis, usually come back with negative results. In our laboratory, we define a positive Western blotting when patient sera show at least four bands for IgG class antibodies, two for IgM class antibodies or two for IgG associated with one $\operatorname{IgM}$ band. This standardization favors sensitivity at the expense of specificity. All the cases de- 
scribed in Brazil obligatorily presented positive Western blotting at the beginning of LDLS or during follow-up (2).

The immunoenzymatic assay (ELISA) usually has a good correlation with Western blotting; however, its sensitivity is lower than that of Western blotting. The ELISA titers observed in Brazil are lower and tend to normalize rapidly during the clinical evolution. In Brazil, ELISA is positive in $46.6 \%$ of the cases diagnosed up to the first 3 months after disease onset, mainly for IgM class antibodies. In the group of patients with more than 3 months of evolution, the ELISA test was positive in $65.5 \%$ of the cases, with a predominance of $\mathrm{IgG}$-dependent responses $(12,13,18,49)$.

The frequency of clinical cases with positive serology, whether ELISA or Western blotting, increases with the performance of sequential serological tests. It is equally important to observe the modification of the Western blotting pattern during follow-up, a relevant laboratory finding that confirms the diagnosis of LDLS (2). The antigen used in serological assays is B. burgdorferi $\mathrm{G} 39 / 40$, an American strain initially isolated from the I. scapularis tick.

The specificity of ELISA was tested by

Table 1. Serology for Borrelia burgdorferi (ELISA) in infectious and rheumatic diseases in Brazilian patients.

\begin{tabular}{llll}
\hline Disease & N & \multicolumn{1}{c}{ IgM } & IgG \\
\hline Normal control & 84 & $2(2.3 \%)$ & $1(1.1 \%)$ \\
Syphilis & 41 & $6(12.5 \%)^{*}$ & $8(56 \%)^{*}$ \\
Leptospirosis & 48 & $6(12.5 \%)^{*}$ & $4(8.3 \%)$ \\
Tuberculosis & 18 & $1(5.5 \%)$ & 0 \\
Malaria (Plasmodium vivax) & 20 & $1(5 \%)$ & 0 \\
Chagas' disease & 30 & $1(3.3 \%)$ & 0 \\
Leprosy & 45 & 0 & $1(2.2 \%)$ \\
Visceral leishmaniosis & 20 & 0 & $5(25 \%)^{*}$ \\
Lupus erythematosus & 31 & $1(3.2 \%)$ & 0 \\
Scleroderma & 82 & $2(2.4 \%)$ & $8(9.7 \%)^{*}$ \\
Rheumatoid arthritis & 35 & $8(22.8 \%)^{*}$ & $2(5.7 \%)$ \\
Juvenile rheumatoid arthritis & 32 & 0 & $1(3.2 \%)$ \\
Spondyloarthropathy & 28 & $1(3.2 \%)$ & $1(3.2 \%)$ \\
\end{tabular}

Data are reported as the number of patients with percent in parentheses. ${ }^{*} \mathrm{P}<0.05$ compared to normal control (chi-square test). analysis of some infectious diseases (syphilis, leptospirosis, tuberculosis, malaria, Chagas' disease, visceral leishmaniosis, and leprosy) and rheumatic diseases (systemic lupus erythematosus, rheumatoid arthritis, juvenile rheumatoid arthritis, and spondyloarthropathy). Cross-reactivity was observed with syphilis, leptospirosis, visceral leishmaniosis, systemic scleroderma, and rheumatoid arthritis (Table 1) (13).

Other laboratory exams can show abnormalities such as transitory elevation of transaminases, high levels of circulating IgE antibodies, hypergammaglobulinemia, and the presence of antinuclear antibodies. Interestingly, in tests that indicate the presence of an acute inflammatory process such as tests for reactive $\mathrm{C}$ protein and erythrocyte sedimentation rate, mucoproteins are altered only in $25 \%$ of patients (13). Inflammatory myositis is a rare condition observed in approximately $5 \%$ of patients with LDLS, and in the presence of this clinical suspicion it is necessary to analyze serum aldolase and creatine phosphokinase. Additional procedures include electromyography and muscle biopsy.

CSF analysis is very important, and should also be done in the presence of few symptoms such as meningismus or lesion of peripheral cranial nerves. The CSF shows a slight increase of mononuclear cells, in general less than 200 cells $/ \mathrm{mm}^{3}$, and discrete elevation of protein levels. Magnetic nuclear resonance may help identify cranial nerve thickness, barrier breaking, or lesion of brain white matter in chronic conditions. Electromyography is helpful to diagnose peripheral nerve lesion or inflammatory myositis. Evoked potential and audiometry are complementary procedures used to confirm cranial neuritis. The analysis of joint fluid from patients with arthritis due to LDLS exhibits inflammatory characteristics with an increase in neutrophils. Eventually, when the serum analysis for B. burgdorferi antibodies is negative and the clinical hypothesis for LDLS is very strong, we can per- 
form tests in CSF or synovial fluid samples, since antibody positivity is higher at inflammatory sites.

The study of autoimmunity features in LDLS, including the anti-nuclear antibodies, anti-bovine brain extract, and anti-rabbit nucleus caudatus extract, has demonstrated the presence of autoantibodies in the sera of LDLS patients $(13,50)$. Interestingly, sera from patients with rheumatic fever reveal crossreactivity with $B$. burgdorferi antigens. Furthermore, sera of patients with LDLS and rheumatic fever recognize the same epitopes present in rabbit nucleus caudatus extract, suggesting that spirochetes and streptococcus may share common epitopes, explaining the clinical similarities of these diseases (50).

\section{Treatment of Lyme disease-like syndrome}

The same drugs used to treat $\mathrm{LD}$ have been employed to treat Brazilian patients with LDLS. However, due to the high frequency of recurrence, we have adopted a prolonged schedule of antibiotic treatment. Follow-up of 25 patients revealed that $10(40 \%)$ presented relapsing episodes mainly occurring in patients with a late diagnosis of the disease (more than 3 months after disease onset), despite treatment with antibiotics (doxycycline or amoxicillin) for at least 15 days (13).

Another sequential study of 19 patients who started with neurological involvement of LDLS (Shinjo SK, Marchiori P, Gauditano G, Costa IP, Oliveira ACP, Bonoldi VLN, Mantovani E, Yoshinari NH, unpublished results), followed up for a mean period or 42.8 months, showed that $14(73.6 \%)$ presented at least one episode of recurrence months or years after LDLS onset.

In an attempt to prevent disease recurrence, we have suggested the use of doxycycline, $100 \mathrm{mg}$ twice a day, or amoxicillin, $500 \mathrm{mg}$ four times a day, for 3 or 4 weeks, when erythema migrans and general symptoms are present during the acute stage of the disease. If arthritis is present in the early stage, alone or associated with erythema migrans, the same antibiotic schedule is maintained for at least 2 months. In the presence of neurological manifestations, the first option is the introduction of ceftriaxone, $2 \mathrm{~g} /$ day, or crystalline penicillin, 24 million U/day, given intravenously for at least 2 weeks, followed by an additional 2 or 3 months of therapy with doxycycline or amoxicillin. During intravenous antibiotic administration, sometimes the Jarisch-Herxheimer reaction is observed, but the treatment must be continued carefully.

Another important question is the existence of co-infection with another tick-borne diseases. There are few studies concerning tick-borne diseases like babesiosis, ehrlichiosis, bartonellosis, rickettsiosis other than Brazilian spotted fever in Brazil. We have recently shown that patients with LDLS present a high incidence of serum antibodies against Babesia bovis, suggesting that the tick transmitter of LDLS could also carry Babesia (51). When the fever is very high (more than $40^{\circ} \mathrm{C}$ ) and is accompanied by important hematological findings such as anemia, reduced platelet numbers or evidence of peripheral hemolysis, the hypothesis of co-infection with Babesia should be considered, and serology for this blood parasite should be performed. If the test is positive, treatment with clindamycin and chloroquine is recommended. Unfortunately, no adequate standardized laboratory tests for co-infections of human patients are currently available in Brazil.

Antibiotics are not efficient for the treatment of post-Lyme syndrome, equally present in Brazilian LDLS patients, or to ameliorate chronic fatigue or fibromyalgia-like symptoms. However, the relapsing disease episodes must be treated, as proposed previously, since the use of antibiotics has proved to be useful to shorten and alleviate recurrent attacks, but new episodes are not prevented by treatment. 
Table 2. Diagnostic criteria of Lyme disease-like syndrome adopted by the Discipline of Rheumatology, Hospital das Clínicas, Faculdade de Medicina, Universidade de São Paulo.

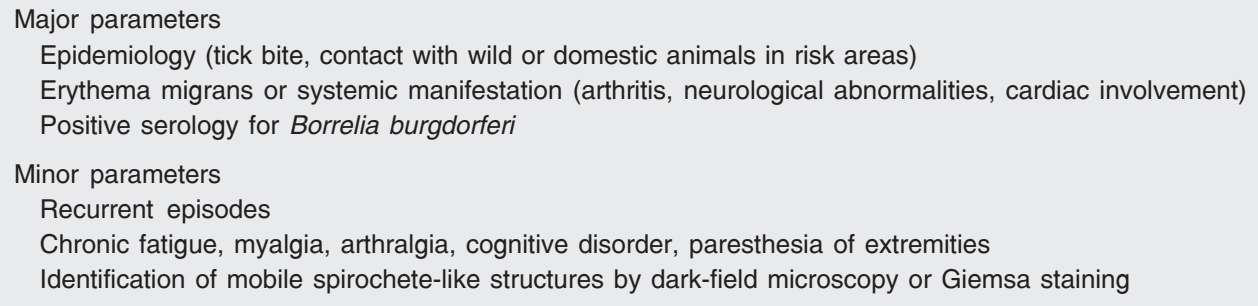

\section{Diagnostic criteria}

The diagnostic criterium of LDLS adopted at the Discipline of Rheumatology of Hospital das Clínicas, Faculdade de Medicina, Universidade de São Paulo, is similar to that employed at the Centers for Disease Control in Atlanta, GA, USA. It is based on the presence of clinical manifestations (erythema migrans or at least one of the systemic complications) associated with positive serology for B. burgdorferi (G 39/40 strain) obtained by indirect ELISA or Western blotting. Additionally, we introduced minor parameters to help clinicians to think about this emerging tick-borne disease in Brazil (Table 2).

Cases are defined as positive in the presence of 3 major parameters or 2 major and 2 minor parameters.

\section{Perspectives and conclusions}

LDLS or LDIS seems to be a new tick- borne disease found in Brazil, which is confused with LD described in the Northern hemisphere. This emerging clinical entity has been progressively recognized by different medical specialists, mainly neurologists, and nowadays the number of monthly serological exams done in our Laboratory is as high as 150 .

LDLS is becoming a serious public health problem, because the number of suspected cases is growing fast and many Brazilian physicians are not yet prepared to diagnose this zoonosis. This difficulty is explained in part by the fact that LDLS has many characteristics differing from those of LD which have taken many years of research to elucidate. Despite many unsolved questions, we assumed that disclosure of some answers obtained in our Laboratory over the last 15 years could help physicians to understand LDLS and consequently help patients to benefit from the dissemination of this knowledge.

\section{References}

1. Steere AC, Malawista SE, Snydman DR, Shope RE, Andiman WA, Ross MR, et al. Lyme arthritis: an epidemic of oligoarticular arthritis in children and adults in three Connecticut communities. Arthritis Rheum 1977; 20: 7-17.

2. Yoshinari NH, Bonoldi VLN, Barros-Battesti DM, Schumaker TTS. Doença de Lyme-símile no Brasil. Rev Bras Reumatol 1999; 39: 57 58.

3. Abel IS, Marzagao G, Yoshinari NH, Schumaker TT. Borrelia-like spirochetes recovered from ticks and small mammals collected in the Atlantic Forest Reserve, Cotia county, State of São Paulo, Brazil. Mem Inst Oswaldo Cruz 2000; 95: 621-624.
4. Costa IP, Bonoldi VL, Yoshinari NH. Search for Borrelia sp. in ticks collected from potential reservoirs in an urban forest reserve in the State of Mato Grosso do Sul, Brazil: a short report. Mem Inst Oswaldo Cruz 2002; 97: 631-635.

5. Yoshinari NH, Barros PJ, Gauditano G, Fonseca AH. Report of 57 cases of Lyme-like disease (LLD) in Brazil. Arthritis Rheum 1999; 43 (Suppl): 188.

6. Benesi FJ, Birgel EH, Paganelli CH, Yoshinari HN, Gregory L, Athayde PF. Lyme disease in cattle in Brazil. Vector Ecol Newsletter 1995; 26: 5-6.

7. Barros-Battesti DM, Yoshinari NH, Bonoldi VL, De Castro GA. Para- 
sitism by Ixodes didelphidis and I. Ioricatus (Acari: Ixodidae) on small wild mammals from an Atlantic Forest in the State of São Paulo, Brazil. J Med Entomol 2000; 37: 820-827.

8. Yoshinari $\mathrm{NH}$, de Barros PJ, Bonoldi VL, Ishikawa M, Battesti DM, Pirana S, et al. Outline of Lyme borreliosis in Brazil. Rev Hosp Clin Fac Med São Paulo 1997; 52: 111-117.

9. Costa IP, Bonoldi VL, Yoshinari NH. Perfil clínico e laboratorial da doença de Lyme-símile no Estado de Mato Grosso do Sul: análise de 16 pacientes. Rev Bras Reumatol 2001; 41: 142-150.

10. Yoshinari NH, Steere AC, Cossermelli W. A review of Lyme disease. AMB Rev Assoc Med Bras 1989; 35: 34-38.

11. Azulay RD, Azulay-Abulafia L, Sodre CT, Azulay DR, Azulay MM. Lyme disease in Rio de Janeiro, Brazil. Int J Dermatol 1991; 30: 569-571.

12. Yoshinari NH, Barros PJ, Cruz FC, Oyafuso LK, Mendonça M, Baggio D, et al. Clínica e sorologia da doença de Lyme no Brasil. Rev Bras Reumatol 1992; 32 (Suppl): 57.

13. Yoshinari NH, Barros PJ, Fonseca AH, Bonoldi VL, Barros-Battesti DM, Schumaker TT. Borreliose de Lyme. Zoonose emergente de interesse multidisciplinar. Newslab 1995; 12: 90-104.

14. Yoshinari NH, Gauditano G, Bonoldi VL, Barros PJ, Novaes GS, Simis DR. Síndrome da doença de Lyme-símile e manifestações cutâneas. Rev Bras Reumatol 1999; 39: 65-69.

15. Masters E, Granter S, Duray P, Cordes P. Physician-diagnosed erythema migrans and erythema migrans-like rashes following Lone Star tick bites. Arch Dermatol 1998; 134: 955-960.

16. Barbour AG, Maupin GO, Teltow GJ, Carter CJ, Piesman J. Identification of an uncultivable Borrelia species in the hard tick Amblyomma americanum: possible agent of a Lyme disease-like illness. $J$ Infect Dis 1996; 173: 403-409.

17. Varela AS, Luttrell MP, Howerth EW, Moore VA, Davidson WR, Stallknecht DE, et al. First culture isolation of Borrelia ionestari, putative agent of southern tick-associated rash illness. J Clin Microbiol 2004; 42: 1163-1169.

18. Yoshinari NH, Steere AC, Barros PJ, Cruz FC, Mendonça M, Oyafuso LK. Lyme disease in Brazil: report of five cases. Rev Esp Reumatol 1993; 20 (Suppl): 156.

19. Melo IS, Gadelha AR, Ferreira LC. Estudo histopatológico de casos de eritema crônico migratório diagnosticados em Manaus. An Bras Dermatol 2003; 78: 1269-1277.

20. Bas S, Scieux C, Vischer TL. Different humoral immune response to Chlamydia trachomatis major outer membrane protein variable domains I and IV in Chlamydia-infected patients with or without reactive arthritis. Arthritis Rheum 1999; 42: 942-947.

21. Kuipers JG, Jurgens-Saathoff B, Bialowons A, Wollenhaupt J, Kohler L, Zeidler H. Detection of Chlamydia trachomatis in peripheral blood leukocytes of reactive arthritis patients by polymerase chain reaction. Arthritis Rheum 1998; 41: 1894-1895.

22. Inman RD, Chiu B. Synoviocyte-packaged Chlamydia trachomatis induces a chronic aseptic arthritis. J Clin Invest 1998; 102: 17761782.

23. Gerard HC, Branigan PJ, Schumacher HR Jr, Hudson AP. Synovial Chlamydia trachomatis in patients with reactive arthritis/Reiter's syndrome are viable but show aberrant gene expression. $J$ Rheumatol 1998; 25: 734-742.

24. Beutler AM, Whittum-Hudson JA, Nanagara R, Schumacher HR, Hudson AP. Intracellular location of inapparently infecting Chlamydia in synovial tissue from patients with Reiter's syndrome. Immunol Res 1994; 13: 163-171.

25. Branigan PJ, Gerard HC, Hudson AP, Schumacher HR Jr, Pando J. Comparison of synovial tissue and synovial fluid as the source of nucleic acids for detection of Chlamydia trachomatis by polymerase chain reaction. Arthritis Rheum 1996; 39: 1740-1746.

26. Koehler L, Nettelnbreker E, Hudson AP, Ott N, Gerard HC, Branigan $\mathrm{PJ}$, et al. Ultrastructural and molecular analyses of the persistence of Chlamydia trachomatis (serovar K) in human monocytes. Microb Pathog 1997; 22: 133-142.

27. Nanagara R, Li F, Beutler A, Hudson A, Schumacher HR Jr. Alteration of Chlamydia trachomatis biologic behavior in synovial membranes. Suppression of surface antigen production in reactive arthritis and Reiter's syndrome. Arthritis Rheum 1995; 38: 1410-1417.

28. Nicolson GL, Gan R, Haier J. Multiple co-infections (Mycoplasma, Chlamydia, human herpes virus-6) in blood of chronic fatigue syndrome patients: association with signs and symptoms. APMIS 2003; 111: $557-566$

29. Higuchi ML, Reis MM, Sambiase NV, Palomino SA, Castelli JB, Gutierrez PS, et al. Coinfection with Mycoplasma pneumoniae and Chlamydia pneumoniae in ruptured plaques associated with acute myocardial infarction. Arq Bras Cardiol 2003; 81: 12-22.

30. Baseman JB, Tully JG. Mycoplasmas: sophisticated, reemerging, and burdened by their notoriety. Emerg Infect Dis 1997; 3: 21-32.

31. Higuchi ML, Gois JM, Reis MM, Higuchi-Dos-Santos MH, Diament $\mathrm{J}$, Sousa JM, et al. Co-infection ratios versus inflammation, growth factors and progression of early atheromas. APMIS 2006; 114: 338344.

32. Morgan M, Nathwani D. Facial palsy and infection: the unfolding story. Clin Infect Dis 1992; 14: 263-271.

33. Nijs J, Nicolson GL, De Becker P, Coomans D, De Meirleir K. High prevalence of Mycoplasma infections among European chronic fatigue syndrome patients. Examination of four Mycoplasma species in blood of chronic fatigue syndrome patients. FEMS Immunol Med Microbiol 2002; 34: 209-214.

34. Nicolson GL, Nasralla MY, Haier J, Erwin R, Nicolson NL, Ngwenya R. Mycoplasmal infections in chronic illnesses: fibromyalgia and chronic fatigue syndromes, Gulf War illness, HIV-AIDS and rheumatoid arthritis. Medical Sentinel 1999; 4: 172-175, 191.

35. Haier J, Nasralla M, Franco AR, Nicolson GL. Detection of mycoplasmal infections in blood of patients with rheumatoid arthritis. Rheumatology 1999; 38: 504-509.

36. Johnson S, Sidebottom D, Bruckner F, Collins D. Identification of Mycoplasma fermentans in synovial fluid samples from arthritis patients with inflammatory disease. J Clin Microbiol 2000; 38: 90-93.

37. Eskow E, Adelson ME, Rao RS, Mordechai E. Evidence for disseminated Mycoplasma fermentans in New Jersey residents with antecedent tick attachment and subsequent musculoskeletal symptoms. J Clin Rheumatol 2003; 9: 77-87.

38. Sibilia J, Limbach FX. Reactive arthritis or chronic infectious arthritis? Ann Rheum Dis 2002; 61: 580-587.

39. Schnarr S, Putschky N, Jendro MC, Zeidler H, Hammer M, Kuipers JG, et al. Chlamydia and Borrelia DNA in synovial fluid of patients with early undifferentiated oligoarthritis: results of a prospective study. Arthritis Rheum 2001; 44: 2679-2685.

40. Rocha JL, Pellegrino LN, Riella LV, Martins LT. Acute hemiplegia associated with cat-scratch disease. Braz J Infect Dis 2004; 8: 263266.

41. Podsiadly E, Chmielewski T, Tylewska-Wierzbanowska S. Bartonella henselae and Borrelia burgdorferi infections of the central nervous system. Ann N Y Acad Sci 2003; 990: 404-406.

42. Pirana S, Yoshinari NH, Silveira AM, Bento RF, Bonoldi VL. Serological reactivity to Borrelia burgdorferi, Borrelia afzelli and Borrelia garinii antigens in patients afflicted by peripheral facial paralysis in Brazil. Otol Neurotol 2002; 23 (Suppl): 33. 
43. Lorenzi MC, Bittar RS, Pedalini ME, Zerati F, Yoshinari NH, Bento RF. Sudden deafness and Lyme disease. Laryngoscope 2003; 113: 312-315.

44. Sato MT, Schmitt A, Greboge P, Arana J, Moreira AT. Neurorretinite associada à ceratite intersticial: relato do primeiro caso de doença de Lyme no Estado do Paraná. Rev Bras Oftalmol 2003; 62: 275283.

45. Steere AC, Coburn J, Glickstein L. The emergence of Lyme disease. J Clin Invest 2004; 113: 1093-1101.

46. Grodzicki RL, Steere AC. Comparison of immunoblotting and indirect enzyme-linked immunosorbent assay using different antigen preparations for diagnosing early Lyme disease. J Infect Dis 1988; 157: 790-797.

47. Dressler F, Whalen JA, Reinhardt BN, Steere AC. Western blotting in the serodiagnosis of Lyme disease. J Infect Dis 1993; 167: 392-
400.

48. Centers for Disease Control and Prevention (CDC). Recommendations for preventing the spread of vancomycin resistance. Recommendations of the Hospital Infection Control Practices Advisory Committee (HICPAC). MMWR Recomm Rep 1995; 44: 1-13.

49. Yoshinari NH, Barros PJ, Yassuda P, Baggio D, Steere AC. Estudo epidemiológico da doença de Lyme no Brasil. Rev Hosp Clin Fac Med São Paulo 1992; 47: 71-75.

50. Gauditano G, Bonoldi VL, Hiratsuka RC, Kiss MH, Yoshinari NH. Aspectos imunológicos comuns entre a doença de Lyme e a febre reumática. Rev Bras Reumatol 2000; 40: 1-8.

51. Yoshinari NH, Abrao MG, Bonoldi VL, Soares CO, Madruga CR, Scofield A, et al. Coexistence of antibodies to tick-borne agents of babesiosis and Lyme borreliosis in patients from Cotia county, State of São Paulo, Brazil. Mem Inst Oswaldo Cruz 2003; 98: 311-318. 